IOANNis K. Argyros (Lawton, OK)

\title{
ON THE NEWTON-KANTOROVICH THEOREM AND NONLINEAR FINITE ELEMENT METHODS
}

Abstract. Using a weaker version of the Newton-Kantorovich theorem, we provide a discretization result to find finite element solutions of elliptic boundary value problems. Our hypotheses are weaker and under the same computational cost lead to finer estimates on the distances involved and a more precise information on the location of the solution than before.

1. Introduction. In this study we are concerned with the problem of approximating a locally unique solution of the nonlinear equation

$$
F(x)=0,
$$

where $F$ is a Fréchet differentiable operator defined on an open convex subset $D$ of a Banach space $A$ with values in a Banach space $B$.

The famous Newton-Kantorovich theorem [4, Th. 6, (1,XVIII)] has been used to show existence and uniqueness of exact solutions of equation (1.1). Moreover, a priori and a posteriori estimates can be obtained as a direct consequence of the Newton-Kantorovich theorem. A survey of such recent results can be found in [2].

Tsuchiya [6] used that theorem to show existence of finite element solutions of strongly nonlinear elliptic boundary value problems. However, it can happen that the basic condition in that theorem, the so-called NewtonKantorovich hypothesis given in [4], is violated and still Newton's method converges [1], [2]. That is why we introduced a weaker hypothesis (see Theorem 2.1 that follows) originated in [1] which can always replace the NewtonKantorovich hypothesis used in [4], [6] (see also (3.3)), and under the same computational cost. This way we can use Newton's method to solve a wider

2000 Mathematics Subject Classification: 65L60, 65N12, 65N15, 65N30, 47H17, 49M15.

Key words and phrases: Newton-Kantorovich method/hypothesis, finite element analysis, Fréchet derivative, isomporphism, boundary value problem, discretization of a nonlinear equation. 
range of problems than before. Moreover, finer estimates on the distances involved and a more precise information on the location of the solution are obtained in [1], [2].

Finally, we provide examples of elliptic boundary value problems where our results apply.

2. Convergence analysis. We state the version of our main result in [2] needed in this study (see also [1, p. 132, Case 3 for $\left.\delta=\delta_{0}\right]$ ).

TheOREM 2.1. Let $F: D \subseteq A \rightarrow B$ be a nonlinear Fréchet differentiable operator, where $D$ is open, convex, and $A, B$ are Banach spaces. Assume:

- there exists a point $x_{0} \in D$ such that the Fréchet derivative $F^{\prime}\left(x_{0}\right) \in$ $L(A, B)$ is an isomorphism and $F\left(x_{0}\right) \neq 0$;

- there exist positive constants $\ell_{0}$ and $\ell$ such that the following center Lipschitz and Lipschitz conditions are satisfied:

$$
\begin{gathered}
\left\|F^{\prime}\left(x_{0}\right)^{-1}\left[F^{\prime}(x)-F^{\prime}\left(x_{0}\right)\right]\right\| \leq \ell_{0}\left\|x-x_{0}\right\|, \\
\left\|F^{\prime}\left(x_{0}\right)^{-1}\left[F^{\prime}(x)-F^{\prime}(y)\right]\right\| \leq \ell\|x-y\|,
\end{gathered}
$$

for all $x, y \in D$.

Setting

$$
\eta=\left\|F^{\prime}\left(x_{0}\right)^{-1} F\left(x_{0}\right)\right\|
$$

and

$$
h_{1}=\frac{1}{4}\left(\ell+4 \ell_{0}+\sqrt{\ell^{2}+8 \ell_{0} \ell}\right) \eta,
$$

we further assume

$$
\begin{aligned}
h_{1} & \leq 1, \\
\bar{U}\left(x_{1}, t^{*}-\eta\right) & =\left\{x \in D:\left\|x-x_{1}\right\| \leq t^{*}-\eta\right\} \subseteq D,
\end{aligned}
$$

where $x_{1}=x_{0}-F^{\prime}\left(x_{0}\right)^{-1} F\left(x_{0}\right)$ and $t^{*} \geq \eta$ is the unique least upper bound of the nondecreasing majorizing sequence $\left\{t_{n}\right\}$ given by

$$
t_{0}=0, \quad t_{1}=\eta, \quad t_{n+2}=t_{n+1}+\frac{\ell_{1}\left(t_{n+1}-t_{n}\right)^{2}}{2\left(1-\ell_{0} t_{n+1}\right)} \quad(n \geq 0)
$$

where

$$
\ell_{1}= \begin{cases}\ell_{0} & \text { if } n=0 \\ \ell & \text { if } n>0 .\end{cases}
$$

Then the equation $F(x)=0$ has a solution $x^{*} \in \bar{U}\left(x_{1}, t^{*}-\eta\right)$ and this solution is unique in $U\left(x_{0}, t^{*}\right) \cap D$ if $\ell_{0}=\ell$ and $h_{1}<1$, and in $\bar{U}\left(x_{0}, t^{*}\right) \cap D$ if $\ell_{0}=\ell$ and $h_{1}=1$. If $\ell_{0} \neq \ell$ the solution $x^{*}$ is unique in $U\left(x_{0}, R\right)$ provided that $\frac{1}{2}\left(t^{*}+R\right) \ell_{0} \leq 1$ and $U\left(x_{0}, R\right) \subseteq D$.

Moreover, we have the estimate

$$
\left\|x^{*}-x_{0}\right\| \leq t^{*}
$$


We will simply use $\|\cdot\|$ if the norm involved is understood. Otherwise we will use $\|\cdot\|_{X}$ for the norm on a particular set $X$.

We assume the following:

$\left(\mathrm{A}_{1}\right)$ There exist Banach spaces $Z \subseteq X$ and $U \subseteq Y$ such that the inclusions are continuous, and the restriction of $F$ to $Z$, denoted again by $F$, is a Fréchet differentiable operator from $Z$ to $U$.

$\left(\mathrm{A}_{2}\right)$ For any $v \in Z$ the derivative $F^{\prime}(v) \in L(Z, U)$ can be extended to $F^{\prime}(v) \in L(X, Y)$ and it is:

- locally Lipschitz continuous on $Z$, i.e., for any bounded convex set $T \subseteq Z$ there exists a positive constant $c_{1}$ depending on $T$ such that

$$
\left\|F^{\prime}(v)-F^{\prime}(w)\right\| \leq c_{1}\|v-w\| \quad \text { for all } v, w \in T
$$

- center locally Lipschitz continuous at a fixed $u_{0} \in Z$, i.e., for any bounded convex set $T \subseteq Z$ with $u_{0} \in T$ there exists a positive constant $c_{0}$ depending on $u_{0}$ and $T$ such that

$$
\left\|F^{\prime}(v)-F^{\prime}\left(u_{0}\right)\right\| \leq c_{0}\left\|v-u_{0}\right\| \quad \text { for all } v \in T .
$$

$\left(\mathrm{A}_{3}\right)$ There are Banach spaces $V \subseteq Z$ and $W \subseteq U$ such that the inclusions are continuous. We suppose that there exists a subset $S \subseteq V$ for which the following holds: "if $F^{\prime}(u) \in L(V, W)$ is an isomorphism between $V$ and $W$ at $u \in S$, then there exists an extension $F^{\prime}(u) \in L(X, Y)$ which is an isomorphism between $X$ and $Y$ as well".

To define discretized solutions of $F(u)=0$, we introduce the finitedimensional subspaces $S_{d} \subseteq Z$ and $S_{d} \subseteq U$ parametrized by $d, 0<d<1$, with the following properties:

$\left(\mathrm{A}_{4}\right)$ There exists $r \geq 0$ and a positive constant $c_{2}$ independent of $d$ such that

$$
\left\|v_{d}\right\|_{Z} \leq \frac{c_{2}}{d^{r}}\left\|v_{d}\right\|_{X} \quad \text { for all } v_{d} \in S_{d}
$$

$\left(\mathrm{A}_{5}\right)$ There exists a projection $\Pi_{d}: X \rightarrow S_{d}$ for each $S_{d}$ such that, if $u_{0} \in S$ is a solution of $F(u)=0$, then

$$
\begin{aligned}
& \lim _{d \rightarrow 0} d^{-r}\left\|u_{0}-\Pi_{d} u_{0}\right\|_{X}=0, \\
& \lim _{d \rightarrow 0} d^{-r}\left\|u_{0}-\Pi_{d} u_{0}\right\|_{Z}=0 .
\end{aligned}
$$

We show the following result concerning the existence of locally unique solutions of discretized equations.

TheOREM 2.2. Assume that conditions $\left(\mathrm{A}_{1}\right)-\left(\mathrm{A}_{5}\right)$ hold. Suppose $F^{\prime}\left(u_{0}\right)$ $\in L(V, W)$ is an isomorphism, and $u_{0} \in S$. Moreover, assume $F^{\prime}\left(u_{0}\right)$ can be decomposed into $F^{\prime}\left(u_{0}\right)=Q+R$, where $Q \in L(X, Y)$ and $R \in L(X, Y)$ 
is compact. The discretized nonlinear operator $F_{d}: Z \rightarrow U$ is defined by

$$
F_{d}(u)=\left(I-P_{d}\right) Q(u)+P_{d} F(u)
$$

where $I$ is the identity of $Y$, and $P_{d}: Y \rightarrow S_{d}$ is a projection such that

$$
\lim _{d \rightarrow 0}\left\|v-P_{d} v\right\|_{Y}=0 \quad \text { for all } v \in Y
$$

and

$$
\left(I-P_{d}\right) Q\left(v_{d}\right)=0 \quad \text { for all } v_{d} \in S_{d} .
$$

Then, for sufficiently small $d>0$, there exists $u_{d} \in S_{d}$ such that $F_{d}\left(u_{d}\right)=0$, and $u_{d}$ is locally unique.

Moreover, the following estimate holds:

$$
\left\|u_{d}-\Pi_{d}\left(u_{0}\right)\right\| \leq \ell_{1}\left\|u_{0}-\Pi_{d}\left(u_{0}\right)\right\|
$$

where $\ell_{1}$ is a positive constant independent of $d$.

Proof. The proof is similar to the corresponding one in [6, Th. 2.1, p. 126]. However, there are some crucial differences where the weaker (2.7) is used (needed) instead of the stronger condition (2.6).

STEP 1. We claim that there exists a positive constant $c_{3}$, independent of $d$, such that, for sufficiently small $h>0$,

$$
\left\|F_{d}^{\prime}\left(\Pi_{d}\left(u_{0}\right)\right) v_{d}\right\|_{Y} \geq c_{3}\left\|v_{d}\right\|_{X} \quad \text { for all } v_{d} \in S_{d} .
$$

From $\left(\mathrm{A}_{3}\right)$ and $u_{0} \in S, F^{\prime}\left(u_{0}\right) \in L(X, Y)$ is an isomorphism. Set $B_{0}=$ $\left\|F^{\prime}\left(u_{0}\right)^{-1}\right\|$.

We have

$$
\begin{aligned}
F_{d}^{\prime}\left(\Pi_{d}\left(u_{0}\right)\right) v_{d}= & F^{\prime}\left(u_{0}\right) v_{d}+P_{d}\left(F^{\prime}\left(\Pi_{d}\left(u_{0}\right)\right)-F^{\prime}\left(u_{0}\right)\right) v_{d} \\
& -\left(I-P_{d}\right)\left(-Q+F^{\prime}\left(u_{0}\right)\right) v_{d}
\end{aligned}
$$

Since $-Q+F^{\prime}\left(u_{0}\right) \in L(X, Y)$ is compact, by (2.12) we get

$$
\lim _{d \rightarrow 0}\left\|\left(I-P_{d}\right)\left(-Q+F^{\prime}\left(u_{0}\right)\right)\right\|=0 .
$$

By (2.12) there exists a positive constant $c_{4}$ such that

$$
\sup _{d>0}\left\|P_{d}\right\| \leq c_{4} \text {. }
$$

That is, using (2.7) we get

$$
\left\|P_{d}\left(F^{\prime}\left(\Pi_{d}\left(u_{0}\right)\right)-F^{\prime}\left(u_{0}\right)\right)\right\| \leq c_{0} c_{4}\left\|\Pi_{d}\left(u_{0}\right)-u_{0}\right\| .
$$

Hence, by (2.10) we have

$$
\left\|F_{d}^{\prime}\left(\Pi_{d}\left(u_{0}\right)\right) v_{d}\right\| \geq\left(1 / B_{0}-\delta(d)\right)\left\|v_{d}\right\|
$$

where $\lim _{d \rightarrow 0} \delta(d)=0$, and so $(2.15)$ holds with $c_{3}=B_{0}^{-1} / 2$. 
STEP 2. We shall show

$$
\lim _{d \rightarrow 0} d^{-r}\left\|F_{d}^{\prime}\left(\Pi_{d}\left(u_{0}\right)\right)^{-1} F_{d}\left(\Pi_{d}\left(u_{0}\right)\right)\right\|=0 .
$$

Note that

$$
\begin{aligned}
\left\|F_{d}\left(\Pi_{d}\left(u_{0}\right)\right)\right\| & \leq c_{4}\left\|F_{d}\left(\Pi_{d}\left(u_{0}\right)\right)-F_{d}\left(u_{0}\right)\right\| \\
& \leq c_{4} \int_{0}^{1}\left\|G_{t}\right\| d t\left\|\Pi_{d}\left(u_{0}\right)-u_{0}\right\| \leq c_{4} c_{5}\left\|\Pi_{d}\left(u_{0}\right)-u_{0}\right\|,
\end{aligned}
$$

where

$$
G_{t}=F^{\prime}\left((1-t) u_{0}+t \Pi_{d}\left(u_{0}\right)\right)
$$

and we used

$$
\begin{aligned}
\left\|G_{t}\right\| & \leq\left\|G_{t}-F^{\prime}\left(u_{0}\right)\right\|+\left\|F^{\prime}\left(u_{0}\right)\right\| \\
& \leq c_{0} t\left\|\Pi_{d}\left(u_{0}\right)-u_{0}\right\|+\left\|F^{\prime}\left(u_{0}\right)\right\| \leq c_{5},
\end{aligned}
$$

where $c_{5}$ is independent of $d$.

The claim is proved.

STEP 3. We use our modification of the Newton-Kantorovich theorem with the following choices:

$$
\begin{aligned}
A & =S_{d} \subseteq Z \quad \text { with norm } d^{-r}\left\|w_{d}\right\|_{X}, \\
B & =S_{d} \subseteq U \quad \text { with norm } d^{-r}\left\|w_{d}\right\|_{Y}, \\
x_{0} & =\Pi_{d}\left(u_{0}\right), \\
F & =F_{d} .
\end{aligned}
$$

Notice that $\|S\|_{L(A, B)}=\|S\|_{L(X, Y)}$ for any linear operator $S \in L\left(S_{d}, S_{d}\right)$.

By Step $1, F_{d}^{\prime}\left(\Pi_{d}\left(u_{0}\right)\right) \in L\left(S_{d}, S_{d}\right)$ is an isomorphism. It follows from (2.6) and $\left(\mathrm{A}_{4}\right)$ that for any $w_{d}, v_{d} \in S_{d}$,

$$
\left\|F_{d}^{\prime}\left(w_{d}\right)-F_{d}^{\prime}\left(v_{d}\right)\right\| \leq c_{1} c_{4}\left\|w_{d}-v_{d}\right\|_{Z} \leq c_{1} c_{2} c_{4} d^{-r}\left\|w_{d}-v_{d}\right\|_{X}
$$

Similarly, using $(2.7)$ and $\left(\mathrm{A}_{4}\right)$ we get

$$
\left\|F_{d}^{\prime}\left(w_{d}\right)-F_{d}^{\prime}\left(\Pi_{d}\left(u_{0}\right)\right)\right\| \leq c_{1} c_{2} c_{4} d^{-r}\left\|w_{d}-x_{0}\right\|_{X}
$$

Hence the assumptions are satisfied with

$$
\ell=c_{1} c_{2} c_{3}^{-1} c_{4} \quad \text { and } \quad \ell_{0}=c_{0} c_{2} c_{3}^{-1} c_{4} .
$$

From Step 2, we may take sufficiently small $d>0$ such that $\left(\ell_{0}+\ell\right) \eta \leq 1$, where

$$
\eta=d^{-r}\left\|F_{d}^{\prime}\left(\Pi_{d}\left(u_{0}\right)\right)^{-1} F_{d}\left(\Pi_{d}\left(u_{0}\right)\right)\right\|_{X} .
$$

That is, the assumption $h_{1} \leq 1$ is satisfied. 
Hence for sufficiently small $d>0$ there exists a locally unique $u_{d} \in S_{d}$ such that $F_{d}\left(u_{d}\right)=0$ and

$\left\|u_{d}-\Pi_{d}\left(u_{0}\right)\right\|_{X} \leq 2 d^{r} \eta \leq 2 c_{3}^{-1}\left\|F_{d}\left(\Pi_{d}\left(u_{0}\right)\right)\right\|_{Y} \leq 2 c_{3}^{-1} c_{4} c_{5}\left\|u_{0}-\Pi_{d}\left(u_{0}\right)\right\|_{X}$.

It follows that (2.14) holds with $\ell_{1}=2 c_{3}^{-1} c_{4} c_{5}$.

That completes the proof of the Theorem.

\section{Concluding remarks and applications}

REMARK 3.1. In general

$$
\left.c_{0} \leq c_{1} \quad \text { (i.e., } \ell_{0} \leq \ell\right)
$$

and $\ell / \ell_{0}$ can be arbitrarily large, where $\ell$ and $\ell_{0}$ are given by (2.26) [1], [2].

If $\ell=\ell_{0}$ our Theorem 2.2 reduces to Theorem 2.1 in [6, p. 126].

Otherwise our condition $h_{1} \leq 1$ is weaker than the corresponding one in [6] using the Newton-Kantorovich hypothesis, famous for its simplicity and clarity:

$$
h=2 \ell \eta \leq 1
$$

(see [4], [1]). That is,

$$
h \leq 1 \Rightarrow h_{1} \leq 1
$$

but not necessarily vice versa, unless $\ell_{0}=\ell$.

As already shown in [2], finer error estimates on the distances $\| u_{d}-$ $\Pi_{d}\left(u_{0}\right) \|$ and a more precise information on the location of the solution are provided here and under the same computational cost since in practice the evaluation of $c_{1}$ requires that of $c_{0}$.

Note also that our parameter $d$ will be smaller than the corresponding one in [6], which in turn implies that fewer computations and smaller dimension subspaces $S_{d}$ are used to approximate $u_{d}$. This observation is important in computational mathematics [1].

The above observations suggest that all results obtained in [6] can be improved if rewritten with weaker $h_{1} \leq 1$ instead of stronger $h \leq 1$.

However, we do not attempt this here (leaving this task to the motivated reader). Instead we provide examples of nonlinear problems already reported in [6] where finite element methods apply along the lines of our theorem above.

EXAmple $3.2([6])$. Find $u \in H_{0}^{1}(J)$ and $J=(b, c) \subseteq \mathbb{R}$ such that

$$
\langle F(u), v\rangle=\int_{J}\left[g_{0}\left(x, u, u^{\prime}\right) v^{\prime}+g\left(x, u, u^{\prime}\right) v\right] d x=0
$$

for all $v \in H_{0}^{1}(J)$, where $g_{0}$ and $g_{1}$ are sufficiently smooth functions from $J \times \mathbb{R} \times \mathbb{R}$ to $\mathbb{R}$. 
ExAmple $3.3([6])$. For the $N$-dimensional case $(N=2,3)$ let $D \subseteq \mathbb{R}^{N}$ be a bounded domain with a Lipschitz boundary. Then consider the problem: find $u \in H_{0}^{1}(D)$ such that

$$
\langle F(u), v\rangle=\int_{D}\left[q_{0}(x, u, \nabla u) \cdot \nabla v+q(x, u, \nabla u) \cdot v\right] d x=0
$$

for all $v \in H_{0}^{1}(D)$, where $q_{0}, q: D \times \mathbb{R} \times \mathbb{R}^{N} \rightarrow \mathbb{R}$ are sufficiently smooth functions.

REMARK 3.4. Since equations (3.4) and (3.5) are in divergence form, their finite element solutions are defined in a natural way. Finite element methods applied to nonlinear elliptic boundary value problems have also been considered by other authors [3], [5]. Finally, more details on Examples 3.2 and 3.3 can be found in [6].

\section{References}

[1] I. K. Argyros, Computational Theory of Iterative Methods, Stud. Comput. Math. 15, Elsevier, New York, 2007.

[2] -, On a class of Newton-like methods for solving nonlinear equations, J. Comput. Appl. Math., to appear; doi: 10.1016/j.cam.2008.08.042.

[3] M. Feistauer and A. Ženišek, Finite element solution of nonlinear elliptic problems, Numer. Math. 50 (1987), 471-475.

[4] L. V. Kantorovich and G. P. Akilov, Functional Analysis in Normed Spaces, Pergamon Press, Oxford, 1982.

[5] J. Pousin and J. Rappaz, Consistency, stability, a priori and a posteriori errors for Petrov-Galerkin methods applied to nonlinear problems, Numer. Math. 69 (1994), 213-231.

[6] T. Tsuchiya, An application of the Kantorovich theorem to nonlinear finite element analysis, ibid. 84 (1999), 121-141.

[7] T. Tsuchiya and I. Babuška, A priori error estimates of finite element solutions of parametrized strongly nonlinear boundary value problems, J. Comput. Appl. Math. 79 (1997), 41-66.

Department of Mathematics Sciences

Cameron University

Lawton, OK 73505, U.S.A.

E-mail: iargyros@cameron.edu

Received on 18.2.2008;

revised version on 26.11.2008 
